\title{
Du XVIe siècle à la Révolution Quelques perspectives générales
}

\section{Philippe Jarnoux et Dominique Le Page}

\section{(C) OpenEdition}

\section{Édition électronique}

URL : http://journals.openedition.org/abpo/1673

DOI : $10.4000 /$ abpo.1673

ISBN : 978-2-7535-1484-3

ISSN : 2108-6443

Éditeur

Presses universitaires de Rennes

Édition imprimée

Date de publication : 20 décembre 2001

Pagination : $31-57$

ISBN : 978-2-86847-674-6

ISSN : 0399-0826

\section{Référence électronique}

Philippe Jarnoux et Dominique Le Page, « Du XVle siècle à la Révolution Quelques perspectives générales », Annales de Bretagne et des Pays de l'Ouest [En ligne], 108-4 | 2001, mis en ligne le 20 décembre 2003, consulté le 21 avril 2019. URL : http://journals.openedition.org/abpo/1673; DOI : $10.4000 / a b p o .1673$ 


\title{
Du XVI ${ }^{\mathrm{e}}$ siècle à la Révolution Quelques perspectives générales
}

\author{
Philippe JARNOUX \\ Maître de conférences en histoire moderne, CRBC-Université de Brest \\ Dominique Le PAGE \\ Maître de conférences en histoire moderne, CRHMA-Université de Nantes
}

Pendant les trois siècles de l'époque moderne, l'histoire de la Chambre des comptes est faite d'une succession de crises, d'adaptations et de transformations encore mal connues mais qui révèlent implicitement les glissements progressifs des rapports de force politiques dans la province et dans le royaume. Notre propos, qui se veut modeste - et provisoire - visera seulement ici à dégager les grandes lignes de l'évolution du personnel, des règlements et des attributions de la cour.

Plusieurs périodes méritent d'être distinguées. À un $\mathrm{XVI}^{\mathrm{e}}$ siècle fait d'agitations politiques, d'incertitudes, de débats et d'implication forte de la Chambre dans les conflits que connaît la Bretagne, succède un premier $\mathrm{XVII}^{\mathrm{e}}$ siècle apparemment plus calme, avant que Louis XIV et Colbert n'imposent de nouveaux changements. La Chambre voit alors son personnel augmenter et ses fonctions décliner aux profits des intendants, des États provinciaux ou du parlement et le XVIII ${ }^{\mathrm{e}}$ siècle confirmera ce déclin d'une Chambre désormais confinée à un contrôle de plus en plus formel des finances. Fondamentalement, les trajectoires de la Chambre des comptes et du Parlement paraissent diamétralement opposées et le destin de la cour nantaise n'est sans doute pas inintéressant pour qui veut scruter les ressorts profonds de l'évolution institutionnelle de la province de Bretagne dans le royaume de France.

\section{La Chambre des comptes (XvI ${ }^{\mathrm{e}}$ siècle-1 ${ }^{\mathrm{re}}$ moitié du XVII ${ }^{\mathrm{e}}$ siècle) ${ }^{1}$}

\section{Des effectifs en hausse}

À la fin de la période ducale, la Chambre des comptes de Bretagne comptait deux présidents, cinq maîtres et auditeurs, neuf clercs et secré-

1. Cette première partie a été rédigée par Dominique LE PAGE, la seconde par Philippe JARNOUX. 
taires (dont deux assumaient alternativement la charge de greffier), un procureur et un personnel annexe composé d'un garde des livres qui faisait office de concierge et d'un huissier. Ce personnel resta presque inchangé jusqu'à la fin du règne de François $\mathrm{I}^{\mathrm{er}}$, les seules modifications ayant été introduites par Charles VIII qui institutionnalisa dès 1492 l'office de trésorier et payeur ${ }^{2}$ et créa une seconde charge d'huissier. Comme dans le reste du royaume, il fallut attendre la seconde moitié du siècle pour assister aux principales mutations ${ }^{3}$. C'est ainsi qu'Henri II procéda aux véritables premières créations en introduisant trois nouveaux offices de maîtres, deux en 1555 et un en octobre 1558. Il fut imité, de façon plus radicale, par Charles IX qui rendit la fonction de greffier autonome en $1569^{4}$, imposa un dixième office d'auditeur en 1570 et surtout doubla le personnel de la Chambre en 1572. Henri III poursuivit sur la lancée de son prédécesseur en imposant tour à tour un office d'avocat général ${ }^{5}$, six charges de correcteur (quatre en 1576, deux en 1588), trois offices de maîtres (un en 1575 et deux en 1588), deux offices d'auditeurs et trois d'huissiers (dont un premier huissier). Passée cette grande vague de créations, les règnes d'Henri IV et de Louis XIII paraissent plus modestes avec l'introduction d'une nouvelle charge de maître en 1598 dans le contexte de règlement du passif ligueur et la création le 17 avril 1626 de 4 offices de correcteurs convertis en 4 charges d'auditeurs - puis en mai 1632, celle de deux mâ̂tres, de deux correcteurs et de deux auditeurs supplémentaires et enfin en 1644, celle d'un deuxième office d'avocat général. À la fin du règne de Louis XIII, on obtient ainsi une Chambre composée de 4 présidents, 23 mâ̂tres, 2 correcteurs, 28 auditeurs, 1 procureur général, deux avocats généraux, 1 premier huissier, sept huissiers, 2 greffiers en chef, 1 garde des livres et un payeur des gages.

À ce personnel, il faut ajouter les officiers qui avaient droit d'entrée à la Chambre à savoir principalement les deux trésoriers de France et généraux des finances et les deux trésoriers généraux ${ }^{6}$. La date d'intégration de

2. Signe d'une volonté de contrôler l'institution, la charge fut confiée à Tanguy de la Gaubertière, secrétaire de Charles VIII.

3. La rareté des créations d'offices par François I ${ }^{\text {er }}$ amène à se demander si, dans la phase de passage de l'union personnelle à l'union définitive de la Bretagne au Royaume, la monarchie ne fut pas contrainte, même implicitement, de respecter non seulement les institutions en place mais aussi le nombre d'officiers les composant.

4. Cette création faisait suite à une demande de la Chambre qui voulait, dès 1564 , qu'une telle transformation ait lieu afin de permettre un meilleur travail, l'alternance des greffiers provoquant une mauvaise tenue des différents registres.

5. Une première tentative pour créer un tel office avait été faite en 1558 par Henri II qui en avait pourvu Jean Boulomer. Le projet n'avait pas abouti du fait de l'opposition du procureur général Guillaume de Francheville qui avait fourni au roi la somme correspondant à la valeur de l'office. En 1575, les choses se passèrent plus simplement puisque Guillaume de Francheville acquit pour lui l'office d'avocat général et céda sa charge de procureur à son fils Jean, Arch. dép. de Loire-Atlantique, B 58 f ${ }^{\circ}$ 169-170.

6. Ils étaient considérés comme faisant partie du corps de la Chambre et bénéficiaient de la distribution de jetons d'argent à l'avènement des rois comme les autres membres du personnel. 
ces catégories au corps des gens des comptes n'est pas connue avec certitude $^{7}$. Pour la première citée, en l'absence de mention de ce droit dans la lettre d'institution du premier général des finances en Bretagne en 1491, on peut penser que cette évolution se fit au cours des années 1530 quand les pouvoirs des généraux furent fortement réduits ${ }^{8}$. La provincialisation de ces officiers ainsi que l'échec de la tentative de création d'un bureau des finances en 1577 durent accentuer le processus d'intégration. Pour les trésoriers généraux, le cas est un peu différent. Comptables soumis à la juridiction de la Chambre, ils étaient dans une position inférieure par rapport à celle-ci. Leur droit d'entrée au Bureau avait surtout pour but de permettre, par leur comparution régulière, un examen rapide de leur gestion ainsi que celle des officiers qui dépendaient d'eux.

Si l'on revient sur les grandes périodes de création d'offices, on doit constater qu'elles n'ont rien pour étonner et qu'elles coïncident avec les moments de difficultés financières de la monarchie, confrontée soit à la guerre extérieure sous Henri II et Louis XIII, soit à la guerre civile sous Charles IX et Henri III. La Chambre ne constitua à chaque fois que l'un des secteurs d'application d'une politique plus vaste qui affecta toutes les institutions de la province et dont on retrouve des échos dans d'autres parties du royaume. Ainsi, les créations relativement réduites de Henri II à la Chambre s'inscrivent-elles dans un contexte qui voit en Bretagne le doublement du nombre de receveurs du fouage - avec la mise en place des receveurs alternatifs -, l'instauration des présidiaux et la création d'un parlement permanent dont le personnel est fixé en 1553 à 4 présidents et 40 conseillers. De même en 1575 quand Henri III se " contenta " d'un nouvel office de maître et d'avocat général à la Chambre, il créa au parlement la chambre criminelle de la Tournelle, ce qui entraîna la mise en vente de deux nouvelles charges de président et de 12 offices de conseillers. Tout fut fait à chaque fois pour tirer parti au maximum des différentes ressources offertes par la province pour alimenter les caisses royales.

Analyser les créations d'offices selon leur seule dimension financière serait pourtant réducteur et reviendrait à en masquer les enjeux, notamment sur le plan des pouvoirs de la Chambre et de son rôle dans la province face aux États et au parlement, aspects sur lesquels nous reviendrons $^{9}$, mais également sur le plan social. Jusque dans les années 1570 à tout le moins, on constate que la vente des charges s'accomplit sans difficulté. Ainsi en 1572, quand le personnel fut doublé, on assista, malgré les réticences des officiers en place, à une véritable course à l'office qui provoqua le recours à des enchères entre les différents prétendants et obligea le roi - pour la seule fois dans l'histoire de la Chambre - à introduire une charge de maître surnuméraire, son candidat ayant été pris de vitesse par

7. Dans le cas de la Bourgogne à la fin du Xve siècle, les généraux faisaient l'acquisition d'un office de maître.

8. P. HAMON, L'argent du roi, les finances sous François ${ }^{\text {er }}$, Paris, CHEFF, 1994.

9. Cf. infra l'article consacré à la crise des années 1560 . 
des personnalités locales. La mise sur le marché de nouveaux offices répondait donc à une attente des élites bretonnes et des régions voisines qui désiraient, en s'introduisant dans l'institution nantaise, concrétiser une réussite et acquérir les privilèges octroyés aux membres d'une cour souveraine. Par la même occasion le pouvoir espérait, dans un contexte de plus en plus incertain, obtenir le soutien des gens fortunés dont il satisfaisait ainsi les ambitions en matière d'honneur et de dignité ${ }^{10}$.

Si après 1572, les choses devinrent plus difficiles, ce n'est pas tant par manque de demande - les offices créés trouvant tous rapidement des acquéreurs - mais par incapacité de la monarchie à imposer sa volonté. Henri III, victime des innovations de son prédécesseur et de la contestation de son pouvoir, dut revoir à plusieurs reprises ses ambitions à la baisse. Ainsi les quatre correcteurs créés en 1576 ne furent-ils admis dans l'institution qu'en 1583; plus sérieusement, en 1581, il échoua à imposer dix officiers de robe longue et il dut se contenter de deux huissiers supplémentaires. De même les innovations de 1588 furent-elles réduites ${ }^{11}$ par rapport au projet initial qui envisageait d'instaurer deux présidents, 12 mâ̂tres, 12 nouveaux auditeurs, 4 correcteurs, 1 huissier de premier degré, 4 d'un rang secondaire, 1 garde-livre. Dans chacun de ces cas, le souverain se heurta à une opposition formée des États de Bretagne hostiles à la multiplication des offices, des parlementaires engagés avec la Chambre dans un conflit de compétences et qui voyaient dans toute augmentation de ses effectifs un risque de plus grand interventionnisme de sa part, des gens des comptes eux-mêmes enfin, réticents à partager les épices entre un nombre de plus en plus élevé de personnes.

En acceptant de renoncer à ses projets, Henri III réussit à obtenir des compensations comme en 1588 où, pour la première fois semble-t-il, les membres de la Chambre lui versèrent 30000 écus pour le prix des offices non créés : ce faisant, tout en récoltant dans l'immédiat les profits attendus, il modifia les termes du débat entre la monarchie et les catégories aisées de la province puisque la satisfaction de ses demandes vint non plus de la création de nouveaux offices qui lui auraient permis de donner une suite favorable aux ambitions de familles locales et donc d'élargir les bases de son pouvoir mais par la consolidation des privilèges des gens en place assurés contre paiement de ne pas subir la concurrence de nouveaux individus. Il contribua ainsi tout à la fois à nourrir les frustrations ${ }^{12}$, à fermer

10. Acheter un office dans un contexte de guerres religieuses pouvait apparaître aussi comme une forme de solidarité à l'égard de l'État engagé dans la lutte contre les protestants.

11. Elles entraînèrent, rappelons-le, la création de 2 maîtres, 2 correcteurs, 4 auditeurs et d'un premier huissier, Arch. dép. de Loire-Atlantique, B 62 f $^{\circ} 219$.

12. Des mouvements comparables se produisirent en Bourgogne, $c f$. H. Drouot, Mayenne et Bourgogne, 1587-1596, Paris, 1937. La difficulté de plus en plus grande d'accéder à la Chambre des comptes, mais aussi le blocage des carrières au sein de cette dernière peuvent expliquer certains aspects de la révolte ligueuse en Bretagne qui traduisit une hostilité grandissante des officiers inférieurs à l'encontre des officiers supérieurs. 
l'institution et à accentuer la mainmise des officiers sur les charges qu'ils détenaient.

\section{Des officiers de plus en plus maîtres de leur destin}

L'un des autres intérêts du XVI ${ }^{\mathrm{e}}$ siècle est de placer l'historien devant une situation mouvante où l'officier cherche de plus en plus à se rendre maître de sa charge et y parvient, au fil des décennies, de moins en moins mal et ce bien avant l'instauration de la paulette en 1604. Quand on analyse les processus de nomination, on voit en effet différents acteurs intervenir au cours de la période étudiée. Le premier d'entre eux était, bien entendu, le roi dont procédaient toutes les créations d'offices, mais dont l'influence est difficile à percevoir directement. On la devine dans le placement de membres de son hôtel ${ }^{13}$ dans des charges de maîtres ou de présidents mais le plus souvent son action était parasitée par différents intermédiaires. Ceux-ci purent disposer officiellement d'un pouvoir de nomination à l'instar d'Anne de Bretagne qui, en vertu du traité conclu avec Louis XII en 1499 à Nantes, contrôla l'administration ducale jusqu'à sa mort en 1514 ou du dauphin Henri qui bénéficia de pouvoirs comparables de 1540 à 1547. Ils purent agir de façon plus officieuse comme Catherine de Médicis dont l'intervention permit notamment l'institution de Jean Coutel comme maître des comptes en $1572^{14}$ ou comme les chanceliers et le personnel gravitant autour de leurs bureaux - notamment les notaires et secrétaires du roi - qui étaient au premier rang pour intervenir en faveur de leurs proches. Parmi les personnalités influentes, il faut citer également les gouverneurs et les Grands de la province : en dehors des Laval dont le rôle est étudié plus loin ${ }^{15}$, on peut mentionner le duc de Montpensier - gouverneur de 1569 à 1582 - qui plaça son intendant Nicolas Coutureau à la présidence de la Chambre ${ }^{16}$ et bien entendu le duc de Mercour qui, à partir de 158917, s'attribua, grâce aux troubles de la Ligue, le droit de pourvoir aux offices

13. On peut citer ainsi à titre d'exemple Jean-François de Cardonne sous Charles VIII ou Gilles de la Pommeraie sous François I ${ }^{\text {er }}$. Le roi se servait aussi souvent des offices pour se libérer d'une dette ou faire payer une pension : ainsi les 2000 écus dus par Pierre Francheville pour son office de maître furent-ils remis au seigneur du Pranay, panetier ordinaire d'Henri II et ambassadeur au Danemark en août 1558, Arch. dép. de LoireAtlantique, B $54 \mathrm{f}^{\circ} 234$.

14. Et peut-être la promotion des Meneust et des Godet au sein de l'institution. Catherine de Médicis s'était vu octroyer d'importants revenus en Bretagne comme ceux de la prévôté de Nantes ou des domaines comme celui de Fougères. Guy Meneust fut un temps son procureur en Bretagne.

15. Cf. infra l'article d'Antoine PACAULT.

16. Celui-ci fut rappelé à l'ordre pour son absentéisme à plusieurs reprises et bénéficia de lettres royales l'autorisant à servir en priorité le gouverneur. Le duc de Montpensier intervint également probablement pour favoriser la carrière de Vital de Contour qui fut trésorier et receveur général de Bretagne puis maître des comptes.

17. Avant cette date, on ne trouve qu'un serviteur proche de Mercœur à la Chambre des comptes, sans qu'on sache si le gouverneur est intervenu pour l'y faire entrer : il s'agit du payeur Mathurin de L'Enfantdieu. 
dans l'institution qui lui était restée fidèle à Nantes ${ }^{18}$. Tout en respectant la clause de style selon laquelle toutes les nominations ne se faisaient qu'à titre de commission ${ }^{19}$ en attendant l'avènement d'un roi catholique, Mercœur en profita pour favoriser la carrière d'hommes qui lui étaient dévoués soit en les faisant entrer dans l'institution comme le Lorrain Jean de Rambouillet ${ }^{20}$ et le Nantais Mathieu de Brénezay ${ }^{21}$, soit en facilitant leur promotion à l'instar de Jean Fourché ou de Jean de la Tullaye qui passèrent, grâce à son appui, du rang d'auditeur à celui de maître. Tous ces changements furent ensuite avalisés par Henri IV lors des négociations de paix; on doit cependant constater que Mercœur ne chercha pas à reconstituer à Nantes une Chambre des comptes complète et qu'il ne multiplia pas les créations d'offices alors que le ralliement de la majorité des maîtres et des présidents à Henri IV l'aurait pourtant rendu nécessaire ${ }^{22}$. Mieux encore, les promotions au rang de maître à Nantes se firent en profitant de décès d'officiers survenus à Rennes, le meilleur exemple étant fourni par Jean Fourché qui, après avoir été promu, demanda et obtint, pour légaliser sa position, à se voir attribuer la charge du royaliste Charles Godet qui venait de décéder ${ }^{23}$. À Nantes comme à Rennes ${ }^{24}$, malgré les divergences idéologiques, ligueurs et royalistes restèrent attachés au nombre d'officiers tel qu'il existait au début de la crise en 1589. Jusqu'au bout, les usages qui voulaient qu'une charge ne fût déclarée vacante que par mort, résignation ou forfaiture du titulaire furent donc respectés, limitant de ce fait la liberté d'action du gouverneur ${ }^{25}$ - et du roi.

Parmi les autres acteurs intervenant dans les processus de nomination, il faut citer aussi bien entendu les officiers eux-mêmes. Au fil du XVI ${ }^{\mathrm{e}}$ siècle,

18. Dès 1589, une Chambre des comptes royaliste s'était constituée à Rennes.

19. Comme dans le parlement ligueur, les nominations furent faites d'abord à titre de commission pour respecter les prérogatives régaliennes puis devinrent de plus en plus des nominations à caractère définitif où le gouverneur agissait en son nom propre.

20. Arch. dép. de Loire-Atlantique, B $63 \mathrm{f}^{\circ} 151$.

21. Ibidem, $\mathrm{f}^{\circ} 198$.

22. On constate ici une évolution parallèle à celle du parlement ligueur étudié par Charles-Antoine CARDOT, Le parlement de la Ligue en Bretagne, 1590-1598, Rennes, 1964, t. I, p. 131.

23. Jean Fourché s'était vu attribuer d'abord l'office d'Étienne Le Franc seul officier royaliste que Mercœur s'était permis de destituer. Il préféra donc prendre un office véritablement vacant. Jean de La Tullaye et Aimé Adam qui avaient été promus maîtres sans que cela corresponde à la libération d'un office à Rennes ne furent, quant à eux, pas remplacés dans leur charge d'auditeurs. Jean de Rambouillet présente un cas particulier : il avait été nommé maître par Mercœur après la mort de Jean Gautier à Rennes mais celuici avait eu le temps de résigner sa charge en faveur de son gendre Joseph de la Marqueraye et l'office n'était donc pas vacant. Fait du hasard ou non, Rambouillet disparut de la Chambre en 1598 et ne fut pas remplacé. Les autres promotions à Nantes se firent dans le cadre de résignations entre officiers ligueurs.

24. Après la mort de Charles Godet, les gens des comptes de Rennes " éteignirent " son office ainsi que celui de François de Bruc, qui venait également de décéder, Arch. dép. de Loire-Atlantique, B $602 \mathrm{f}^{\circ}$ 276, mention du 18 septembre 1592.

25. L'influence des gouverneurs de la première moitié du XvII siècle reste à étudier ainsi que de façon plus générale celle des Grands de la province de Bretagne. 
en effet, ces derniers bénéficièrent du développement de la vénalité ${ }^{26}$ et des progrès de l'hérédité. La Bretagne suivit sur ce point l'évolution du royaume ${ }^{27}$ avec l'introduction de la vénalité publique en 1523 et l'affinement progressif des procédures de résignation : résignations simples, résignations à survivance dès le règne de Charles VIII, introduction de la clause des quarante jours dans les années 1530, application de la clause du tiers denier une première fois en 1568 - mais sans qu'on sache véritablement l'accueil qui lui fut réservé - puis de façon plus systématique sous Henri III ${ }^{28}$. Avant même la création de la paulette et en dépit des volontés répétées du pouvoir de réduire régulièrement le nombre de ses agents ${ }^{29}$ voire de remettre en cause la vénalité, les officiers acquirent donc des avantages importants ${ }^{30}$ et ce d'autant plus que la monarchie usa de moins en moins souvent de l'arme de la destitution.

Le souverain qui y eut le plus recours est François $\mathrm{I}^{\mathrm{er}}$ qui, dans le cadre de la réorganisation des finances des années 1530, contraignit quatre officiers de la Chambre à se démettre de leur charge du fait soit de leur incapacité à l'exercer ${ }^{31}$, soit des malversations réelles ou supposées qu'on leur reprochait ${ }^{32}$. Après son règne et jusque dans la première moitié du XVI ${ }^{\mathrm{e}}$ siècle, les destitutions furent exceptionnelles et ne relevèrent jamais d'opérations à grande échelle : elles visèrent des officiers qui, à titre individuel, avaient commis des délits dans l'exercice de leur fonction comme le président Georges Le Mignot ${ }^{33}$ suspendu en 1587 et le maître Jean Melou en 1650

26. La situation est mal connue pour la fin du Moyen Âge même si la vénalité était déjà sans doute en vigueur, J. KERHERVE, L'État breton aux XIVe et XVe siècles, les ducs, l'argent et les hommes, Paris, 1986.

27. R. MousNIER, La vénalité des offices sous Henri IV et Louis XIII, Paris, 1971.

28. Le premier membre de la Chambre à avoir profité de cette possibilité fut le maître Marc de Barbère, ancien notaire et secrétaire royal, en mars 1577 : contre le versement de 400 écus dans les caisses royales, il obtint le droit de résigner son office en faveur de la personne de son choix sans payer de somme supplémentaire et en échappant à la règle des quarante jours. En cas de décès prématuré, ses héritiers pouvaient disposer de sa charge, Arch. dép. de Loire-Atlantique, B 59 f 29.

29. Cette volonté s'exprima de façon très nette dans les années 1560 - cf. infra l'article sur la crise des années 1560 - et encore sous Henri III qui s'engageait en 1588 à ramener le nombre d'officiers à ce qu'il était au début du Xvie siècle. Les problèmes financiers ne permirent pas à ces projets d'aboutir.

30. Ce sont les individus et pas encore les familles qui furent les acteurs principaux des transactions pendant la majeure partie du Xvi ${ }^{\mathrm{e}}$ siècle; à partir de l'instauration du tiers denier, les parents de l'officier jouèrent toutefois un rôle croissant.

31. C'est le cas des présidents Jean de Plédran et Guillaume Loisel le jeune, Cf. D. LE PAGE, Finances et politique en Bretagne au début des temps modernes, 1491-1547, Paris, CHEFF, 1997, p. 208 et suivantes.

32. C'est le cas du premier président Jean Parajau qui fut contraint de céder son office pour solder ses comptes d'ancien trésorier général et de Guillaume Loisel l'aîné.

33. Arch. dép. de Loire-Atlantique, B 62 f $^{\circ}$ 183-184. Les raisons de sa suspension restent encore à éclaircir. Ce que l'on sait c'est que Le Mignot s'opposa à ce que les gens des comptes de Nantes rayent une somme de 1748 écus au compte de Gilles Blandin, receveur d'Auray, ce qui entraîna le renvoi de l'affaire devant le conseil d'État. Celui-ci envoya deux commissaires à Nantes - $\mathrm{M}^{\mathrm{e}}$ François Dolu, conseiller au conseil privé, président 
ou qui s'étaient compromis dans des affaires criminelles comme dans le cas du maître Jean Monneraye, accusé de meurtre et chassé de la Chambre en $1648^{34}$. Les deux autres moments qui auraient pu être propices à des mutations du personnel furent étrangement calmes : ainsi au cours des années 1560, aucun officier, à l'exception peut-être du payeur des gages des gens des comptes Jean Avril ${ }^{35}$, ne fut mis à l'écart pour son adhésion au protestantisme alors qu'en 1562 un document ${ }^{36}$ signalait qu'un président, un mâ̂tre et deux auditeurs étaient gagnés aux idées nouvelles. L'impossibilité, pour le moment, d'identifier tous les individus concernés avec certitude rend difficile l'interprétation de cette différence entre la Chambre et le parlement de Rennes où treize offices tenus par des protestants furent supprimés en 1570 : les mutations du personnel - par mort ou résignation de charges - avant le déclenchement - tardif -, de la répression ${ }^{37}$, la rétractation de quelques officiers ${ }^{38}$, le probable caractère passager de leur ralliement aux idées protestantes, une solidarité plus grande de corps ${ }^{39}$ peuvent en l'état actuel des connaissances être retenus comme des hypothèses de travail pour expliquer ces évolutions dissemblables ${ }^{40}$. La période de la Ligue ne provoqua pas elle non plus de bouleversements : malgré les anathèmes lancés de part et d'autre, malgré la virulence du vocabulaire employé, les deux camps ne procédèrent pas à des destitutions massives de leurs adversaires $^{41}$ et se figèrent très vite sur leurs positions attendant l'évolution des évé-

en la Chambre des comptes de Paris et $\mathrm{M}^{\mathrm{e}}$ Denis Barthéllémy, maître ordinaire en la Chambre - qui confirmèrent la décision de la Chambre, suspendirent Le Mignot et le condamnèrent à payer la somme contestée. Du fait du déclenchement de la crise ligueuse et de son décès, Le Mignot ne put jamais retrouver sa charge.

34. Pour les cas de Mélou et de Monneraye, $c f$. Hervé Audrain, La Chambre des comptes de Bretagne au temps de la Fronde, mémoire de maîtrise, dactyl., Université de Nantes, 2000.

35. Celui-ci ne prêta pas le serment de catholicité exigé par le procureur de la Chambre en 1569 et trois ans, plus tard, en 1572, le général des finances demandait aux gens des comptes d'instituer quelqu'un pour faire le paiement de leurs gages, ce qui permet de supposer qu'Avril avait été suspendu de sa charge, Arch. dép. de Loire-Atlantique, B 588 f $^{\circ} 234$.

36. Il s'agit d'une lettre du secrétaire Pierre Cornulier au duc d'Étampes, gouverneur de Bretagne, Dom MoRICE, Mémoires pour servir de preuves à l'histoire ecclésiastique et civile de Bretagne, Paris, 1746, t. III, col. 1324.

37. Ce n'est qu'à partir de 1569 que la chasse aux officiers protestants fut vraiment engagée dans la province.

38. C'est le cas de Pierre Ménardeau qui, en mai 1569, avoua avoir été protestant mais affirma être revenu dans le giron de l'Église catholique, Arch. dép. de Loire-Atlantique, B $586 \mathrm{f}^{\circ} 216$.

39. Cette solidarité joua en faveur du payeur Jean Avril dont les gens des comptes mirent du temps à se séparer. Il est vrai qu'ils étaient dépendants de lui pour le paiement de leurs gages.

40. Les gens des comptes poursuivent à la même période tous les receveurs des finances suspectés de protestantisme.

41. Mercœur n'aurait procédé qu'à une seule destitution parmi les officiers royalistes : il s'agit de celle d'Étienne Lefranc. On ne connaît pas les raisons de ce traitement particulier. Cette attitude rejoint celle qui a été décrite par Henri Drouot pour la Bourgogne ou par Stéphane Gal pour le Dauphiné, cf. Grenoble au temps de la Ligue, Étude politique, sociale et religieuse d'une cité en crise (vers 1562-vers 1598), Grenoble, 2000. 
nements. La reconstitution par Henri IV à Nantes en 1598 d'une Chambre des comptes unique réunissant ligueurs et royalistes, permit de faire taire - du moins en apparence - les divisions, fournissant une preuve supplémentaire de la politique d'alliance nouée entre ce souverain et les élites, quel que soit l'engagement passé de celles-ci.

Bénéficiant d'un statut de plus en plus en plus précis, échappant aux menaces de la destitution, les gens des comptes n'eurent pas à subir par ailleurs de contrôle particulièrement sévère lors de leur entrée dans l'institution. Soumis depuis l'origine à une enquête visant à vérifier leurs bonnes vies et mœurs ${ }^{42}$ et à s'assurer qu'eux ou leurs parents n'avaient pas manié de finances sans en rendre compte, ils durent, à partir du règne d'Henri II, prêter un serment de catholicité et jurer de respecter les règlements de la Chambre ${ }^{43}$. On sait aussi qu'ils étaient soumis à un examen en matière de justice et de finances pour vérifier leurs compétences, ce qui put amener la Chambre à recaler certains postulants : ainsi la réception de Guy du Couëdor fut-elle en décembre 1570 retardée d'un an "pendant lequel temps il fera tout debvoir et diligence de s'exercer et faire instruire sur le faict de justice et finances pour audit jour procéder à sadite réception s'il est deu que faire ce doive et qu'il soit trouvé suffisant et cappable de l'exercice d'iceluy ${ }^{44}$ ". Ce cas est toutefois exceptionnel et les réticences à la réception d'un candidat s'expliquent le plus souvent par son âge, ses liens de famille avec un membre de l'institution ${ }^{45}$, l'hostilité des gens des comptes à la création de l'office dont il était pourvu ${ }^{46}$. La Chambre n'exigeait pas de grades universitaires de ses membres - ce qui n'empêche pas bien sûr que l'on rencontre parmi eux des docteurs, des licenciés, des

42. En octobre 1572, le procureur général demandait ainsi à René Hachon si depuis sa réception à l'état d'auditeur - cela s'était passé en 1559 -, il n'avait " esté accusé d'aucun crime ». Hachon répondit que non et ajouta que " lors qu'il fut receu auditeur, il fut recherché exactement et à toutes rigueurs mais nonobstant tous les dires, remontrances du procureur général, il obteint arret à son profit et fut receu ", Arch. dép. de LoireAtlantique, B $588 \mathrm{f}^{\circ} 278$.

43. À titre d'exemple, on peut citer le serment prêté par Aymé Adam le 11 mai 1570 : il jura « de garder les ordonnances et édits du roy, d'exercer diligemment et fidèlement ledit estat, de garder l'ordonnance de 1557 faite par les commissaires venuz céans, de n'allouer aucunes sommes es comptes qu'il examinera mais en faire rapport au bureau pour icelles parties allouer ou reffuser si besoing est et de ne se absenter sans congé de la Chambre ", Arch. dép. de Loire-Atlantique, B 587 f $^{\circ} 183$.

44. Arch. dép. de Loire-Atlantique, B 588 f $^{\circ} 164$. Le décès de Guy du Couëdor avant la fin du délai qui lui avait été fixé ne lui permit pas de se soumettre à un nouvel examen de la Chambre, Arch. dép. de Loire-Atlantique, B 589 f $^{\circ} 300$.

45. L'ordonnance d'Orléans de 1560 interdisait de recevoir dans le même corps deux proches parents mais des entorses furent faites à ce principe en usant de différents arguments : ainsi Guillaume et Pierre de Francheville furent-ils autorisés à siéger ensemble au nom du principe que le second, en tant que maître, était juge alors que l'autre qui était procureur, ne l'était pas. Après la création de deux semestres en 1572, obligation fut faite aux officiers qui avaient des liens de parenté de siéger dans deux ouvertures différentes.

46. Les gens des comptes retardèrent ainsi systématiquement la réception des officiers qui furent nommés dans les années 1570-1580 en s'appuyant sur la promesse qu'avait faite Charles IX en 1572 de supprimer l'office surnuméraire créé en 1572. 
avocats $^{47}$ - et reconnut toujours, sinon privilégia, au moins pour le XVI ${ }^{\mathrm{e}}$ siècle, l'expérience acquise sur le terrain. C'est pourquoi elle accueillit dans ses rangs nombre d'anciens officiers de finances qu'ils fussent receveurs ordinaires, receveurs du fouage ou trésoriers généraux ${ }^{48}$, et qu'elle continua à former elle-même une partie de son personnel, notamment dans les degrés les plus bas de la hiérarchie ${ }^{49}$. Cette importance accordée à l'expérience explique l'attachement d'une partie des auditeurs à la promotion à l'ancienneté et leur hostilité à la vénalité des offices qui les privait progressivement de toute possibilité de promotion ${ }^{50}$.

Les besoins de l'État ainsi que les ambitions des catégories aisées des provinces ligériennes firent pourtant triompher l'argent sur le talent et pour entrer à la Chambre, il fallut être capable de mobiliser des sommes de plus en plus importantes. La rupture, en Bretagne, comme ailleurs se produisit à partir de l'instauration de la paulette en 1604. D'après les indications chiffrées dont nous disposons pour la seconde moitié du XVI ${ }^{\mathrm{e}}$ siècle, les charges de présidents se négociaient autour de 8000 à 10000 l, celles de maîtres autour de 4000 à $6000 \mathrm{l}$ et celles d'auditeurs autour de 2000 à $4000 \mathrm{l}$. Il n'y avait pas encore de prix fixe du fait des enchères qui avaient lieu lors de la création des offices, du fait aussi des différents moments de création de ces derniers, du fait enfin des marchandages entre certains officiers et le pouvoir, les premiers proposant, une fois en place, de payer un supplément dans les caisses royales afin d'obtenir plus de gages ${ }^{51}$. Ces disparités entraînaient des conflits entre les gens des comptes qui manifestaient régulièrement le souci, afin probablement de maintenir la cohésion du corps, qu'à chaque office corresponde une valeur précise $\mathrm{e}^{52}$. Si ces problèmes ne disparurent pas dans la première moitié du XVII ${ }^{\mathrm{e}}$ siècle, l'augmentation sensible du prix des charges les mit cependant au second plan : à titre de com-

47. On peut citer quelques docteurs comme le procureur général Guillaume de Francheville, des licenciés en droit (Golven de Kerchrist, Guillaume Morin, Geoffroy de Callac...). Ce sont les plus gradués que l'on envoyait le plus souvent en délégation vers le roi pour défendre les intérêts de la Chambre.

48. L'intégration de ces derniers, connus pour leur faible formation universitaire, conforte l'idée que de nombreux gens des comptes n'étaient pas passés par l'université.

49. C'est le cas des gardes des livres : ainsi Guillaume Meneust qui voulait résigner à survivance son office en 1568 en faveur de son fils Charles donnait comme argument pour appuyer sa demande " qu'il l'avait retenu et dressé depuis trois ans ". Les auditeurs nouvellement reçus étaient par ailleurs associés à un auditeur plus ancien qui leur apprenait le métier (cas notamment de François de Bruc, Arch. dép. de Loire-Atlantique, B 587 $\mathrm{f}^{\circ}$ 160-161).

50. Cette hostilité s'exprima lors de la crise ligueuse au cours de laquelle les gens des comptes demeurés à Nantes demandèrent à ce que les offices ne fussent pas attribués pour de l'argent. On ne sait pas si Mercœur tint compte ou non de cette demande.

51. Au fil du siècle, ceux-ci correspondirent de moins en moins à la rétribution d'un travail effectué mais à des intérêts proportionnels aux sommes versées au roi. Ce sont les épices qui récompensèrent le zèle quotidien de l'officier.

52. Les sommes versées donnaient droit à des gages et à des privilèges : il était donc important, pour ne pas multiplier les divisions, qu'à chaque degré de l'institution, les officiers fussent placés sur le même pied. 
paraison avec les chiffres mentionnés précédemment, on peut ainsi indiquer qu'au début des années 1650 un office d'auditeur s'achetait entre 30000 et 35000 livres, une charge de maître autour de 60000 livres, une charge de procureur général autour de 150000 l. Les gens des comptes appartenaient donc bien désormais aux élites de la province, sans égaler toutefois "Messieurs du parlement " avec lesquels ils étaient entrés en compétition depuis le $\mathrm{XVI}^{\mathrm{e}}$ siècle.

\section{Une volonté de régularisation du travail des gens des comptes}

En dehors de l'augmentation des effectifs et des progrès de la vénalité, le dernier trait qui marque le $\mathrm{XVI}^{\mathrm{e}}$ siècle et la première moitié du XVII ${ }^{\mathrm{e}}$ siècle est l'effort réalisé par les autorités pour normaliser le travail des gens des comptes. Malgré la création ancienne de la Chambre, on n'a en effet gardé aucun règlement pour le Moyen Âge et il semble donc que ce soit simplement à partir du $\mathrm{XVI}^{\mathrm{e}}$ siècle que les autorités se soient inquiétées de légiférer en la matière. Sans prendre en compte les ordonnances valables pour l'ensemble du royaume, on constate, pour la seule province de Bretagne, la promulgation d'édits importants en 1505, 1513, 1535, 1557, 1581, 1582, $1599^{53}$. La chronologie ne tient pas là non plus du hasard et l'on peut distinguer trois grands moments dans cette œuvre législative. Le premier correspond aux lendemains de l'intégration de la Bretagne au royaume de France où il fallut définir la place de la Chambre dans le nouveau cadre politique et institutionnel : en dehors de Louis XII qui intervint ponctuellement pour interdire aux comptables ayant exercé dans la province de se pourvoir devant les instances parisiennes, Anne de Bretagne, de façon plus systématique, promulgua deux ordonnances - en 1505 et en 1513 - afin de réaffirmer la souveraineté des gens des comptes en matière financière ${ }^{54}$, leur autorité sur le personnel judiciaire et leur rôle de gardiens du domaine. Soucieuse de garantir les privilèges de la Bretagne, ce qui passait pour elle par le maintien de ses institutions, elle élabora ainsi les textes de base de la Chambre qui servirent de références tout au long de l'époque moderne.

Une deuxième série de textes coïncide avec les temps de forte crise financière particulièrement sous François Ir $^{\mathrm{er}}$ et Henri II ainsi qu'à la fin des guerres de la Ligue. À chaque fois, le souci de rétablir l'ordre dans les finances et de soumettre les financiers à un contrôle plus strict amena les autorités à rappeler les règles de reddition des comptes - calendrier, mode de présentation des comptabilités, sanctions à l'égard des contrevenants...-

53. Pour tous ces règlements, l'ouvrage de référence est celui d'ARTuR de la GIBONAYS, Recueil des édits et ordonnances et règlements concernant les fonctions ordinaires de la Chambre des comptes..., Nantes, 1721, auquel nous renvoyons le lecteur.

54. Avec le droit de juger tous les comptables de la province mais aussi tous ceux qui intervenaient dans la gestion de l'hôtel de la reine dont le financement était assuré par des sommes prélevées en Bretagne. Ce pouvoir de contrôler des financiers extérieurs à la province dura jusqu'à la fin du règne de François $\mathrm{I}^{\mathrm{er}}$ puisque les hôtels de la reine Claude et celui du dauphin Henri furent également alimentés par des fonds bretons. 
mais aussi à mieux définir les fonctions des différents officiers de la Chambre afin de faire de ceux-ci des relais plus efficaces de la monarchie. Division des tâches, hiérarchisation du personnel, gestion plus rigoureuse de l'espace de l'institution mais aussi du temps avec la réglementation des ouvertures furent les maîtres mots des réformes engagées qui furent à chaque fois, et c'est un autre point commun, menées voire imposées, par des instances extérieures à la Chambre qu'il s'agisse d'officiers du parlement de Rennes comme le président Guillaume Poyet en 1535 ou de membres de la Chambre des comptes de Paris comme Michel Tambonneau en 1557 ou Maupeou en 1599. À la différence des ordonnances de la première période distinguée, les textes promulgués par François $\mathrm{I}^{\mathrm{er}}$, Henri II et Henri IV concernèrent davantage le personnel - gens des comptes, officiers de finances - que les pouvoirs de la Chambre ${ }^{55}$ : l'État profita à chaque fois de l'urgence financière pour tenter de modifier les comportements.

Le troisième temps des réformes correspond aux règnes de Charles IX et de Henri III et à un moindre degré à celui de Louis XIII. La question des pouvoirs de l'institution revint au premier plan comme sous le règne d'Anne de Bretagne mais cette fois pour définir la place de la Chambre dans le jeu institutionnel au sein de la province. Avec l'instauration d'un parlement permanent en 1554, la Chambre des comptes se retrouva en effet face à un adversaire qui remit progressivement en cause sa souveraineté en matière financière et son droit à juger en première instance des questions domaniales, obligeant le pouvoir royal à se poser en arbitre dans la querelle entre les deux cours. Objet de multiples sollicitations, voyant ses arrêts contestés, la monarchie tergiversa longtemps et ce n'est que sous Henri III qu'une solution durable fut trouvée, là encore après une valse hésitation désordonnée. La première inclination du roi fut de donner raison à la Chambre en élargissant nettement le champ de ses compétences : par un édit de 1581, il lui reconnut la souveraineté en matière domaniale et l'autorisa à se parer du titre de cour des aides et finances. En contrepartie, il obtint la création de dix offices de maître de robe longue au sein de l'institution : le souci de gagner de l'argent n'était donc pas absent mais l'ambition du roi ne se résumait pas à cela puisque son projet, s'il avait abouti, aurait donné des pouvoirs sans précédent à la Chambre. Dès 1582, pourtant, après de nouvelles consultations, le roi annula ses principales concessions ${ }^{56}$ : ainsi sur la question controversée du domaine, il ôta à la Chambre le droit de s'occuper des procès provoqués par les questions de propriété et d'usurpation. La volte-face du monarque s'explique par la pression conjuguée du parlement et des États de Bretagne qui, au nom du respect des privilèges bretons, refusèrent toute extension de la juridiction des gens des comptes et conclurent en

55. Même s'il n'est pas toujours facile de distinguer les deux aspects, l'organisation du travail des gens des comptes ayant toujours des répercussions sur leur pouvoir.

56 . Il dut renoncer par la même occasion aux dix officiers de robe longue qu'il avait voulu créer. 
l'occasion une alliance qui révéla l'hostilité d'une partie des élites de la province à l'idée d'un contrôle efficace des finances et du domaine royal en Bretagne. Ce faisant, ils limitèrent le rôle de la Chambre et, pour cela, le règne d'Henri III apparaît, en l'état actuel des recherches, comme un moment important dans le recul de son influence.

L'effort réglementaire déployé par la monarchie tout au long du XVI ${ }^{\mathrm{e}}$ siècle eut pour conséquence d'aligner progressivement la Chambre des comptes de Bretagne sur le mode de fonctionnement de la Chambre des comptes de Paris et de parachever une évolution institutionnelle amorcée depuis le Moyen Âge puisque les cours des comptes des principautés étaient nées en s'inspirant du modèle parisien ${ }^{57}$. Cette influence se remarque dans l'application en Bretagne de réformes qui avaient été préalablement introduites à Paris : ainsi l'ordonnance d'Anne de Bretagne de 1513 suivait-elle de peu celle de Louis XII de 1511; de même la semestrialisation, qui fut imposée à Nantes en 1572 en liaison avec le doublement du personnel, existait déjà à Paris depuis $1552^{58}$. L'évolution de la titulature des officiers copiait aussi le modèle parisien ${ }^{59}$. Aux trois grandes catégories que constituaient les présidents, les auditeurs et maîtres, les clercs et secrétaires au début du XVI ${ }^{\mathrm{e}}$ siècle se substitua, dans la seconde moitié du siècle, une classification qui, en dessous des présidents, distinguait des maîtres et des auditeurs. Il n'y avait pas là seulement jeu sur les termes mais aussi définition plus précise des tâches des uns et des autres puisque les maîtres qui siégeaient au grand Bureau avec les présidents assumaient une fonction principalement judiciaire alors que les anciens secrétaires étaient chargés presque exclusivement d'auditionner les comptes, d'où le nouveau nom qui leur était attribué.

Face au modèle parisien, l'attitude des gens des comptes de Bretagne fut empreinte de pragmatisme et visa banalement à rejeter ce qui pouvait remettre en cause leur mode traditionnel de fonctionnement et à accepter ce qui entraînait une revalorisation de leur statut. Ainsi les auditeurs furentils hostiles à l'application à Nantes des règles de travail en vigueur à Paris, qui en les reléguant dans des tâches comptables et en les plaçant dans une position inférieure par rapport aux maîtres, entraînaient une dévalorisation de leur office : ils empêchèrent l'application du règlement Poyet en 1535 et, s'ils ne purent s'opposer aux réformes de Tambonneau en 1557, celles-ci ne furent mises en œuvre qu'après une crise grave de l'institution. Le clivage présidents-maîtres/auditeurs devint à partir de cette période

57. La France des principautés, les Chambres des comptes aux XIVe et XV siècles, Colloque de Moulins, Paris, CHEFF, 1996.

58. En juin 1572 quand il s'agit de doubler le personnel à Nantes, les gens des comptes de Paris - qui se qualifiaient de " frères et bien bons amys " de ceux de Nantes - leur envoyèrent un extrait de l'édit fait par le roi en février 1552 pour la création de semestres dans leur compagnie, Arch. dép. de Loire-Atlantique, B 589 f 94.

59. La charge de correcteur fut de la même façon introduite à Nantes en 1576 en reprenant le modèle parisien, alors que la charge ne s'y imposait pas vraiment en raison de la taille réduite de la circonscription financière bretonne. 
une donnée importante de la vie de la Chambre permettant de comprendre une partie des tensions qui s'y développèrent.

Sur d'autres points, les gens des comptes cherchèrent à tirer profit du modèle parisien : ainsi sur le plan des privilèges, ils revendiquèrent les mêmes droits que leurs homologues parisiens, ce qui leur permettait d'espérer non seulement une amélioration de leur condition personnelle mais aussi de pouvoir faire meilleure figure par rapport aux officiers du parlement de Rennes qui disposaient d'un meilleur statut. L'assimilation à l'institution parisienne sur le plan des privilèges visait en outre, à moyen et long terme, à faciliter une assimilation sur le plan des compétences : les gens des comptes de Nantes, à partir de la seconde moitié du XVI ${ }^{\mathrm{e}}$ siècle, ambitionnèrent ainsi ouvertement que la jurisprudence ${ }^{60}$ en vigueur entre la cour des comptes et le parlement de Paris fût appliquée en Bretagne pour définir leurs relations avec les parlementaires rennais.

\section{Évolutions et fonctionnement de Colbert à la Révolution}

Avec le règne de Louis XIV et le ministère de Colbert, la Chambre des comptes connaît à nouveau un certain nombre de réformes réorganisant son fonctionnement habituel, son personnel et ses attributions. La deuxième moitié du XVII ${ }^{\mathrm{e}}$ siècle voit l'institution prendre une forme presque définitive.

\section{Des efforts de remise en ordre}

L'initiative passe d'abord par une surveillance plus rigoureuse des officiers. Les années 1660 sont marquées par la volonté de Colbert de réorganiser et de restreindre la vénalité des offices ${ }^{61}$. Mais cette tentation de réformer, voire de supprimer le système des offices ne résistera pas aux humeurs belliqueuses et aux nécessités financières de Louis XIV. Colbert l'abandonnera définitivement avec la guerre de Hollande et la seconde partie du règne présentera une politique très différente de la monarchie vis-àvis des cours supérieures.

La remise en ordre commence par la réaffirmation des conditions restrictives d'admission aux offices. La déclaration de décembre 1665 et l'édit de juillet 1669 reviennent sur les conditions d'âges et les interdictions liées à la parenté. Ces édits sont répétés à plusieurs reprises et légèrement adaptés ou modifiés. Les âges prescrits dans l'édit de 1665 sont de 40 ans pour les présidents, 30 pour les avocats et procureurs généraux et 27 pour

60. L'influence de la jurisprudence adoptée entre la Chambre des comptes et parlement de Paris se remarque en 1565, quand Charles IX, lors de sa venue en Bretagne, confirma que dans les commissions pour les révisions de compte mises en place en Bretagne, il y aurait comme à Paris un nombre équivalent de parlementaires et de gens des comptes.

61. W. Doyle, "Colbert et les offices ", Histoire, Économies, Sociétés, 2000, t. 19, n 4, p. $469-480$. 
les maîtres, correcteurs et auditeurs ${ }^{62}$. Cependant, après une confirmation en 1672 un autre édit en novembre 1683 abaisse à 25 ans l'âge requis pour les maîtres, correcteurs et auditeurs. De même, en matière de parenté, si on ne revient pas sur les principes généraux, on note parfois quelques adaptations. Initialement, l'édit de juillet 1669 interdisait que pères, fils, frères, oncles, neveux, beaux-pères, gendres et beaux-frères soient reçus simultanément, que les titulaires déjà en place contractent des mariages dans cette parenté prohibée et précisait que, pour les officiers déjà pourvus, les voix de parents ne compteraient que pour une. Mais, dès le 27 décembre 1669 , une déclaration royale lève l'incompatibilité parentale entre deux correcteurs ou deux auditeurs " parce que... dans nos chambres des comptes, les fonctions des auditeurs et des correcteurs sont de telle qualité qu'ils ne se trouvent jamais opiner ensemble ${ }^{63}$ ". Certes ces restrictions sur les âges et la parenté ne sont pas nouvelles. Depuis longtemps, la monarchie les répète sous des formes variées. Ce qui est intéressant ici, c'est la multiplication des interventions dans un laps de temps relativement court, la dureté du ton des édits et des peines prévues. Les textes sur l'âge des officiers sont répétés en 1665, 1669, 1672 et modifiés en 1683; ceux sur la parenté et le vote des parents reviennent en 1669, 1679, 1681 et $1708^{64}$. Les officiers reçus en contravention à ces règles encourent la « nullité des provisions et réceptions " et même la perte de l'office en cas de mariage prohibé (" déclarons l'office du dernier reçu vacant à notre profit ") 65 .

Les édits tentent aussi de contrôler plus étroitement l'octroi de lettres d'honneur que nombre de cours souveraines accordaient sans que les demandes en aient été réellement adressées à Versailles. Sans impact réel sur l'activité des cours et sans portée politique ${ }^{66}$, ces privilèges de vétérance ont toutefois un coût facile à mesurer pour la monarchie puisqu'ils procurent des exemptions diverses à des individus qui, en échange, ne rendent plus aucun service effectif au monarque. Surveiller plus rigoureusement l'octroi de l'honorariat revient à la fois à réaffirmer l'autorité du pouvoir sur les compagnies d'officiers, à leur rappeler que le monarque est la

62. "Nous avons estimé que la réformation principale de la justice consistoit en celle des juges; et qu'il importoit principalement de n'en commettre la dignité qu'a personnes choisies, qui fussent d'une intégrité et capacité éprouvées et d'une maturité d'âge qui pût répondre au public de l'expérience nécessaire pour en bien soutenir l'autorité ", Édit de juillet 1669 portant règlement général pour les offices de judicature du royaume. G. ARTUR de la GiBOnAYs, op. cit., $3^{\mathrm{e}}$ partie, p. 274.

63. G. ARTUR de la GiBONAYs, idem, déclaration du 27/12/1669.

64 . Une déclaration du roi sur le sens à donner au mot "beau-frère " revient encore sur le sujet en 1728, Arch. dép. de Loire-Atlantique, B 100, f ${ }^{\circ} 120 \mathrm{r}$.

65 . Ces menaces restent virtuelles, comme on pouvait s'y attendre. On trouve cependant parfois dans les archives du parquet quelques actes du procureur général critiquant les cumuls ou s'opposant - momentanément - à quelques réceptions d'officiers, mais aucune véritable procédure n'est engagée. Arch. dép. de Loire-Atlantique, B 151.

66. Les lettres accordées à des membres de la Chambre des comptes signalent toujours qu'elles ne donnent pas voix délibérative aux officiers ainsi distingués mais qu'elles ne leur apportent que les privilèges et exemptions. 
seule source de leur autorité ou de leurs privilèges et à faire peut-être quelques infimes économies.

Enfin, dans le même ordre d'idée, par la déclaration de décembre 1665, Colbert s'attache à fixer les prix des offices pour contrecarrer leur tendance permanente à la hausse. Les offices de président sont fixés à 160000 livres (pour le premier président) et 100000 , celui du procureur général à 80000 , ceux de maîtres à 50000, les charges de correcteurs et d'avocat général valent 30000 livres et celles d'auditeur sont établies à $16000^{67}$. Cette fixation participe des projets de suppression de certains offices de Colbert mais vise aussi à limiter les spéculations ou les espoirs de gain importants qui font se précipiter les acheteurs : les prix établis alors ne correspondent guère qu'à 70 ou 80 \% des prix réels pratiqués dans les années 1650 .

Il n'est pas facile de savoir si ces édits ont un impact sur la situation de la Chambre des comptes. Le règlement sur les prix de 1665 semble globalement respecté, si l'on excepte le cas des auditeurs qui contestent le prix très bas de leurs charges. Il en va de même apparemment pour les limites d'âge. Parmi les officiers reçus entre 1645 et 1665, un tiers au moins paraissent en dessous des âges prescrits; en revanche, de 1665 à 1685, les cas de non respect sont très rares $^{68}$. À l'inverse, le respect des règles de parenté est manifestement plus difficile. De 1645 à 1665, des cumuls familiaux se sont produits à 7 reprises; on en trouve 9 cas dans les deux décennies suivantes $^{69}$. Malgré la rigueur apparente de la monarchie, les lettres de dispense existent encore, les règlements ne sont pas toujours bien appliqués... Et la situation se dégradera à nouveau considérablement au XVIII ${ }^{\mathrm{e}}$ siècle.

Parallèlement, la remise en ordre passe par la détermination définitive des privilèges accordés aux officiers. La Chambre a obtenu momentanément en 1659 la noblesse au premier degré pour ses officiers à l'instar du parlement :

«Voulons \& Nous plaist, qu'ils joüissent, eux, leurs Veufves demeurantes en viduité, leur posterité \& lignée, tant mâles que femelles, nais \& à naistre, des mesmes Droits \& Privileges, Immunitez, Rang, Seance \& preéminences, que les autres Nobles de race de Gentils-hommes \& Barons de nostredit Païs;

67. Très sous-évaluées, ces dernières seront portées quelques années plus tard à 20000 , puis 26000 livres. À titre de comparaison, le même texte fixe les prix des offices parlementaires : 90000 à 150000 livres pour les présidents, 50000 à 110000 livres pour les conseillers et 70000 à 150000 livres pour les gens du roi. La différence entre les deux cours souveraines bretonnes est sensible, et seuls les maîtres et les présidents peuvent considérer que leurs charges équivalent à celles du Parlement. Cf. supra, les données évoquées dans la première partie de ce texte.

68. Dans l'attente de la réalisation du catalogue prosopographique complet qui nous permettra de répondre avec assurance à cette question, nous ne pouvons travailler que sur des données partielles. Sur 62 officiers reçus entre 1645 et 1665, on connaît l'âge de 24 : 9 n'ont pas l'âge requis. Après 1665, pour 64 officiers et 49 âges connus, deux seulement sont trop jeunes.

69. Encore n'avons-nous pas tenu compte des liens d'oncle à neveu que nous n'avons pas encore la possibilité de repérer systématiquement; on peut penser que $15 \%$ des officiers environ se trouvent dans des situations de cumul parental. 
qu'ils soient capables de parvenir à tous honneurs, charges \& dignitez, pourveu que lesdits Officiers ayent servy vingt années, ou qu'ils decedent revestus desdits Offices, nonobstant qu'ils ne fussent issus de noble \& ancienne race : Et d'autant que la pluspart des Officiers de nostredite Chambre sont Nobles d'extraction, \& par leur naissance, Nous voulons que ces presentes leur servent d'accroissement d'honneur \& de generosité ${ }^{70}$."

Peut-être est-ce là le résultat de la docilité de la Chambre lors des troubles de la Fronde :

Ils nous ont même donné de si forte \& entieres preuves de leurs inclinations à nostre service depuis l'heureux advenement à la Couronne dont rien n'a iamais esté capable de les détourner dans les derniers troubles \& mouvemens de cét Estat que nous avons sujet de nous loüer de leur obeïssance \& fidélité ${ }^{71} \ldots »$

Mais ce texte a donné lieu à de nombreuses contestations en particulier de la part des États de Bretagne et dans le cadre des procédures de réformation de la noblesse. L'édit sera finalement révoqué par le règlement de 1669 et jamais appliqué. Dorénavant, les gens des comptes se contenteront d'un anoblissement graduel après deux générations ou pour les enfants d'un officier mort en charge et il ne semble pas que la Chambre ait jamais tenté de retrouver cet anoblissement au premier degré. Sur ce problème de l'effet anoblissant des offices des comptes, se greffe, de plus, le vieux conflit qui oppose les auditeurs au reste de la Chambre. Ce sont les auditeurs, majoritairement roturiers, qui auraient le plus à gagner à cet anoblissement alors que nombre de maîtres et présidents sont, de toute façon, déjà nobles, mais ces derniers ne sont pas prêts à se battre pour les premiers. Le débat paraît partiellement réglé avec un édit de 1675 qui réaffirme clairement l'appartenance des auditeurs aux " conseillers du roi " mais les maintient néanmoins dans une position subalterne à l'intérieur de l'institution ${ }^{72}$. Dans la pratique, il ressurgit de temps à autre tout au long du XVIII ${ }^{\mathrm{e}}$ siècle, mais sans avoir la même acuité qu'auparavant ${ }^{73}$.

Cette remise en ordre passe enfin, et peut-être surtout, par l'établissement de nouveaux règlements internes. Depuis le $\mathrm{XVI}^{\mathrm{e}}$ siècle le fonctionnement de la Chambre des comptes a été progressivement ramené à celui de la Chambre de Paris. Mais cette évolution ne s'est pas faite sans

70. Lettres patentes du Roy portant confirmation des privilèges de Messieurs de la Chambre des Comptes de Bretagne, données à Paris au mois d'avril 1659. Arch. dép. de Loire-Atlantique, B 151.

71. Ibidem.

72. C'est du moins ce qu'on considère à Versailles puisque l'édit de 1675 est indiqué dans le préambule du règlement de 1681 comme une des mesures prises pour ramener l'ordre au sein de la Chambre.

73. Le premier exemple de ce débat lancinant a lieu dès 1677 quand les auditeurs prétendent s'opposer à la nomination d'un huissier sous prétexte que le grand bureau (composé des maîtres et présidents) ne les a pas consultés à ce propos. Un arrêt du conseil déboute les auditeurs le 11 janvier 1678 (Arch. dép. de Loire-Atlantique, B 86). Des exemples similaires de conflits internes se retrouvent dans les livres des audiences et des mandements royaux du XVIII siècle. 
difficulté et n'est sans doute que très relative au XVII ${ }^{\mathrm{e}}$ siècle. En 1669 un édit réorganise le fonctionnement de toutes les chambres des comptes du royaume. C'est fondamentalement un règlement technique avec pour but principal d'améliorer ou de réformer quelques points du contrôle des comptes et de supprimer quelques abus " mineurs" (l'usage de simples récépissés à la place de quittances, l'obligation de résidence, la surveillance des épices ou les fonctions des huissiers) ${ }^{74}$. Ce règlement est toutefois mal appliqué à Nantes, son inexécution permet les fraudes et des faux documents réalisés à l'occasion de la réformation de la noblesse amènent la monarchie à envoyer un intendant dresser un procès verbal de l'état des archives. C'est le rapport de cet intendant, Béchameil de Nointel, qui incite Colbert à prévoir un nouveau règlement, particulier à la Chambre bretonne en 1681. Ses origines, la condition de sa mise en place et les considérations évoquées dans le préambule de l'édit sont particulièrement intéressants dans ce qu'ils révèlent de l'image que l'on peut se faire à Paris de la cour nantaise :

" Il [Henri II] en fit un particulier pour celle de Bretagne, par lequel, après avoir donné quelque forme de compagnie supérieure à des officiers auparavant établis par la seule autorité ducale, les fonctions des officiers de cette Chambre qui avoient auparavant été exercées avec quelques confusions, furent réglées suivant l'ordre de leur dignité et préséance... Par l'édit de 1582, la manière de dresser, présenter, juger et apurer les comptes a été établie; et par celui de l'année 1625, les contestations survenües entre notre cour de Parlement et notre Chambre des comptes de Bretagne ont été terminées et assoupies... Nous avons aussi par notre édit de l'année 1669, réprimé et corrigé plusieurs abus... Mais la sagesse de toutes les loix n'a pû prévoir tous les cas particuliers, ni remédier à tous les abus qui se sont introduits par la suite des temps, dans notre Chambre des Comptes de Bretagne; et en ayant été informé par le sieur Bechameil, Maître des Requêtes ordinaire de nôtre Hôtel, par nous commis pour la reformation de nôtre Domaine de Bretagne, Nous aurions mandé les Sieurs Becdelièvre de la Busnelaye, premier président, le Marié Doyen des Maîtres, et Morice, Procureur Général en nôtre Chambre des Comptes de Bretagne, et après qu'ils ont eû communication des avis à Nous envoyes, et qu'il ont été plusieurs fois oüys, par devant le sieur Colbert, Conseiller ordinaire au Conseil Royal, Contrôleur Général de nos Finances, Nous avons resolu de pourvoir à ce qui n'est pas suffisemment expliqué dans les precedentes ordonnances; de renouveller la disposition de celles dont on s'est relâché; d'y ajoûter ce que nous avons estimé nécessaire pour etablir plus d'ordre et de subordination dans la fonction des officiers; et une regle plus certaine dans la forme de dresser, presenter, juger, apurer et corriger les comptes avec les precautions suffisantes pour la conservation des Titres justificatifs de nos Droits, remis dans les Dépôts de nôtre Chambre des Comptes de Bretagne ${ }^{75}$."

74. Édit du roy servant de règlement général pour toutes les Chambres des comptes du royaume, août 1669. G. ARTUR de la GiBONAYS, op. cit., $2^{\mathrm{e}}$ partie, p. 93-99.

75. Édit en forme de règlement pour la chambre des comptes de Bretagne, août 1681. G. ARTUR de la GiBonaYs, op. cit., p. 160-164. 
De fait, ce nouveau règlement revient brièvement sur quelques règles relatives aux comptes et aux actes de foi et hommage ${ }^{76}$ mais s'arrête surtout sur les principes relatifs aux archives, sur les modalités de délivrance d'extraits, sur les dépôts des registres des actes au greffe ou au garde des livres et sur la surveillance de l'ensemble par le procureur général. Les vérifications de Béchameil ont sans doute révélé l'ampleur du laisser-aller qui règne alors dans les dépôts de la Chambre, peut-être l'ampleur des faux et des fraudes et la nécessité d'y mettre fin. Un arrêt du conseil d'État " touchant à l'altération et falsification de quelques titres de la Chambre des comptes " avait d'ailleurs déjà été pris le 12 octobre $1680^{77}$ et la surveillance est probablement rigoureuse pendant quelque temps. Après 1681, la Chambre des comptes semble définitivement stabilisée dans son fonctionnement général et, jusqu'à la Révolution, les modifications ne porteront dorénavant que sur des modalités très spécifiques et ponctuelles.

Si le fonctionnement de la Chambre est désormais fixé, la deuxième moitié du règne de Louis XIV, se caractérise, au contraire de la précédente, par la multiplication du nombre des officiers. Désormais, il n'est plus question de réduire ou de contrôler la vénalité mais bien plutôt d'utiliser le système pour les recettes fiscales qu'il procure à l'État, politique classique et habituelle de l'office utilisé comme expédient financier, en temps de guerre en particulier. Depuis le milieu du XVII ${ }^{\mathrm{e}}$ siècle, la Chambre compte en théorie ${ }^{78}$ 74 officiers. Or, en moins de 20 ans, la monarchie crée 31 nouveaux offices. En 1692, au cœur de la guerre de la Ligue d'Augsbourg, on crée 1 président, 3 maîtres, 2 correcteurs et 2 auditeurs. Avec la guerre de Succession d'Espagne, en 1704, un autre édit entraîne la création de 2 présidents, 4 mâ̂tres, 4 correcteurs et 4 auditeurs auxquels s'ajoutent deux généraux de finances, puis un substitut du procureur. Ces créations ne se limitent pas à la chambre bretonne puisque, au total, 120 nouveaux offices apparaissent alors dans les diverses chambres des comptes du royaume ${ }^{79}$. Après cet afflux massif, seront encore institués 1 maître en 1708, 2 généraux de finances en 1709 et 1 président et 2 maîtres en 1711. Encore ne retenons nous ici que les offices qui vont subsister et qui n'ont pas été rachetés par la Chambre elle-même ou supprimés peu après leur création ${ }^{80}$. Ces créa-

76. Par exemple la nécessité de faire signer tout arrêt par le président ou l'annulation de tous les dons de "fruits de mal foy " faits depuis 1675 (les fruits de mal foy sont les biens saisis en raison d'un défaut de foy et hommage).

77. Arch. dép. de Loire-Atlantique, B 86.

78. En théorie seulement, car dans la pratique, certains cumulent parfois pendant quelque temps plusieurs offices, d'autres sont morts et pas encore remplacés... Tous les offices ne sont pas toujours pourvus.

79. 18 à Rouen, 14 à Nantes, 14 à Metz, 6 à Pau, 16 à Dijon, 16 à Grenoble, 12 à Aix, 16 à Dôle et 8 à Blois. Nous ne retenons pas dans ces chiffres les fonctions subalternes de commis écrivains, huissiers et procureurs. Arch. dép. de Loire-Atlantique, B 151. Il serait intéressant de savoir combien seront effectivement pourvus et maintenus.

80. La complexité des créations et suppressions d'offices est parfois extrême : on fait feu de tout bois pour obtenir de l'argent des officiers. L'exemple des offices de greffier est significatif. De deux, leur nombre est d'abord porté à quatre en 1695, puis ramené 
tions d'offices sont bien sûr autant d'expédients financiers qui ne rapportent pas toujours beaucoup à l'État mais qui coûtent cher aux officiers déjà en place. La Chambre s'efforce en effet de limiter ces créations et de les racheter. Fondamentalement, on sait bien que ces créations d'offices et ces augmentations de gages s'apparentent à des emprunts forcés et, au total, la Chambre déboursera énormément et ces affaires financières donneront lieu à des conflits durables jusqu'aux années 1730, entre la chambre et son payeur des gages par exemple.

\section{Un grignotage progressif des fonctions}

Les innovations administratives du règne de Louis XIV vont, d'autre part, singulièrement réduire et remettre en cause certaines des attributions de la Chambre. La politique de Colbert qui cherche à remettre de l'ordre dans les ressources traditionnelles de la monarchie est à l'origine de deux grandes œuvres de réformation, celle de la noblesse et celle du domaine royal dans lesquelles le rôle et la situation de la Chambre des comptes sont très différents.

Jean Meyer a déjà longuement présenté la réformation de la noblesse, son organisation et ses implications ${ }^{81}$. La Bretagne a obtenu qu'elle soit réalisée par une commission et non par un intendant, mais la commission mise en place est constituée uniquement de parlementaires et non d'officiers des comptes. Pourtant, sur un tel thème, leur présence n'eut peut-être pas été incongrue; étant les gardiens des archives les plus importantes, ils auraient pu prétendre y participer ${ }^{82}$. De fait, la Chambre des comptes ne joue un rôle dans cette réformation que parce que les familles doivent fréquemment s'adresser à elle pour obtenir copie des archives qu'elle conserve et qui attestent de leur noblesse. La Chambre a ainsi des possibilités d'influer sur des décisions individuelles mais pas sur la marche générale de la réformation et, manifestement, aucun de ses membres n'a été jugé suffisamment compétent et respecté pour participer à la commission ${ }^{83}$.

à deux par arrêt du conseil du 25 juin 1697. Le sieur Bazillais possède alors les deux offices (un par semestre) et obtient de les unir en l'échange d'un paiement de 10000 livres. Mais un édit de décembre 1699 supprime l'office de greffier. Bazillais proteste, arguant des dépenses qu'il a engagées deux ans plus tôt et est finalement autorisé à les relever en 1701 pour la somme de 19000 livres! Il conservera les deux offices jusqu'en 1718, avant d'en résigner un à son fils. Arch. dép. de Loire-Atlantique, B 153.

81. Cf. J. MeYER, La noblesse bretonne au XVIII siècle, Paris, EHESS, chap. 2, p. 29-60.

82. Il faut noter toutefois que parmi les 17 membres de la commission, un certain nombre ont des liens avec la chambre, parce qu'ils y ont siégé ou qu'ils y ont eu des parents.

83. Le choix d'une commission composée exclusivement de parlementaires relève aussi toutefois d'une stratégie politique bien entendue. Le pouvoir de "nuisance " du Parlement et des États apparaît bien plus grand que celui de la Chambre et en agissant ainsi, on affaiblit les éventuelles contestations ultérieures. À l'inverse, choisir des gens des comptes aurait pu être considéré comme une provocation ou un affront par une partie de la noblesse bretonne, d'autant plus que tous les membres de la Chambre ne sont pas - loin de là - d'une noblesse ancienne ou irréprochable. 
Il en va tout autrement pour la réformation du Domaine. Nous sommes ici dans un champ d'activité privilégié des gens des comptes et le projet de constitution d'une commission souveraine en 1659 s'est heurté à un tollé et une protestation vive de toutes les institutions de la province. Un arrêt du conseil du 26 mai 1660 révoquant les déclarations précédentes rappelle en particulier l'opposition des États qui y voient une infraction aux privilèges de la province, une surcharge pour " les sujets de Sa Majesté " et soulignent que " toute la fonction d'icelle se fait à présent sans aucuns frais par la Chambre des Comptes et les Juges ordinaires de ladite province avec plus d'utilité pour Sa Majesté ${ }^{84}$ ". Il s'agit toutefois d'une victoire momentanée. En 1664, la Réformation des Bois et Forêts de Bretagne, qui relevaient également de la surveillance de la Chambre des comptes, est opérée par des officiers extérieurs et, en 1670, la monarchie créera finalement une nouvelle commission pour connaître des usurpations sur le Domaine Royal. Établie à Rennes et composée à nouveau de parlementaires, elle intègrera néanmoins des gens des comptes, tels le procureur général de la Chambre à partir de 1673. À nouveau les États s'opposent à cette commission, non pas pour défendre les intérêts de la Chambre des comptes mais parce qu'elle constitue, à leurs yeux, une menace pour les privilèges de la province. En 1671, ils offrent 2 millions de livres en échange de la suppression de cette commission qu'ils obtiendront finalement en 1674 avec la nomination de commissaires issus cette fois de la Chambre des comptes. Avec les commissaires des comptes, toutefois la réformation n'avance guère et il faudra un nouvel arrêt du conseil en 1678 - très critique envers la Chambre des comptes - pour que les choses s'accélèrent enfin. Le Roi y désigne 8 maîtres et présidents et l'opération sera enfin menée à bien et terminée vers 1686. Entre temps toutefois, en 1680, la commission d'intendant accordée à Béchameil lui donne aussi un droit de regard sur la Réformation et inquiète beaucoup de gens dans la province. Si la Chambre des comptes a réussi à conserver en partie son autorité en ce domaine, elle le doit surtout aux craintes des États de voir le pouvoir central intervenir trop directement plus qu'à sa propre activité, par ailleurs apparemment peu appréciée par la Monarchie et presque mise sous tutelle par le regard du commissaire du roi.

Les difficultés de la mise en place de la réformation du Domaine ne sont sans doute pas sans conséquences sur l'avenir de la Chambre et suggèrent peut-être au monarque la création d'un bureau de finances dans la province. C'est un autre épisode important où le pouvoir et les attributions de la Chambre sont contestés. L'édit du 20 avril 1694 qui le met en place se justifie par l'augmentation du travail des trésoriers de France mais on sent bien que l'argumentation est de pure forme. Le bureau comprendra les deux trésoriers de France généraux de finances jusqu'alors rattachés à la Chambre des comptes qui deviendront présidents, huit trésoriers, un avo-

84. Arrêt du Conseil du 26 mai 1660, dans G. Artur de la GiBonaYs, op. cit., $1^{\text {re }}$ partie, p. 106-109. 
cat et un procureur du roi, un greffier, deux receveurs, trois huissiers ${ }^{85}$ et six procureurs. Établi à Rennes puis transféré à Vannes, ce bureau a des attributions qui recouvrent très largement des fonctions jusqu'alors dévolues à la Chambre des comptes et amoindrissent considérablement son rôle. L'opposition à la création de ce bureau est d'abord le fait des deux généraux de finances déjà en place, Heron et Dondel. Directement mise en cause, la Chambre ne peut néanmoins qu'enregistrer l'édit le 22 mai 1694 mais elle y joint des remontrances, fait assez exceptionnel de sa part sous le règne de Louis XIV ${ }^{86}$. Toutefois, l'opposition la plus vive et la plus régulière vient du Parlement et surtout des États de Bretagne qui y voient une atteinte grave aux privilèges bretons. Ils en font un des articles de leur cahier de remontrances au roi après les sessions de 1695, 1697 et 1699. L'attitude de la Chambre des comptes, même si elle est résolue dans son opposition, est variable selon les années. Après les remontrances, qui ont sans doute peu d'impact, la Chambre demande des précisions sur la délimitation des fonctions du bureau, essayant ainsi de le vider des activités qui lui sont concurrentes et de diminuer au maximum ses pouvoirs ${ }^{87}$. Plus systématiquement, elle offre de racheter les charges créées. La Chambre des comptes offre de " payer les sommes auxquelles montent la finance principale, le marc d'or, les deux sols pour livre, frais et loyaux coûts des offices dudit bureau suivant la liquidation qui en sera faite et la somme de 30000 livres et les 2 sols pour livre pour ledit office de notre procureur près l'intendant ". En avril et juin 1696, la monarchie crée encore trois nouveaux offices $^{88}$, mais, finalement, les propositions de rachat des gens des comptes seront acceptées et en septembre 1700, le bureau de finances est supprimé et les offices remboursés.

En six ans, il n'a pas eu une grande activité mais sa brève existence est néanmoins symbolique. Elle atteste du type de mesures " contraires aux privilèges bretons " que la monarchie en guerre est prête à prendre (et qu'elle eut pu imposer probablement) mais aussi du peu d'estime dont jouit indirectement la Chambre des comptes. Un simple bureau de finances pourrait la remplacer dans ses fonctions habituelles. Et son maintien tient certes aux protestations bretonnes mais aussi au bon vouloir royal.

85. Il s'agit d'un receveur payeur des gages, d'un receveur payeur des épices, droits et amendes, d'un huissier garde-meuble et de deux huissiers collecteur des finances. ARTUR de La GiBOnAIS, op. cit., $1^{\text {re }}$ partie, p. 109.

86. Il faut rappeler que depuis la déclaration du 24 février 1673, toutes les cours supérieures du royaume sont contraintes à l'enregistrement pur et simple des édits royaux, les remontrances ne peuvent venir que dans un second temps. La déclaration du 15 septembre 1715 rétablira les cours dans le droit de remontrance préalable.

87. À l'automne 1697, par exemple, la Chambre envoie une députation à Versailles (composée du premier président Becdelièvre et de deux maîtres, Bernard et Mosnier) pour solliciter un règlement entre elle et le bureau de finances. Arch. dép. de Loire-Atlantique, registres d'audience, 1697-1711.

88. Ces deux derniers offices seront rachetés en 1698 par les trésoriers de France généraux de Finances et ne seront jamais créés. Arch. dép. de Loire-Atlantique, B 91. 
Avec l'installation à demeure d'un intendant en 1689 un nouveau regard administratif immédiat prend place dans le jeu politique provincial. Les commissions d'intendants leur donnent en effet un pouvoir sur les finances, ce qui les met en contact, voire en opposition, avec la Chambre des comptes. Parmi les premiers intendants, Béchameil connaît bien la Chambre pour avoir été chargé en 1680 d'en inspecter les archives et la présentation qu'il fait du système fiscal breton dans son célèbre rapport de 1698 montre qu'il est particulièrement attentif à ces questions ${ }^{89}$. Dans l'ensemble, le regard des intendants est sans complaisance pour la Chambre ainsi qu'en atteste la correspondance entre Ferrand et Mellier ${ }^{90}$. Cela tient autant à des relations personnelles délicates qu'à des interférences de pouvoir. L'exemple du conflit qui oppose en 1711 le premier président de la Busnelais à Ferrand est des plus révélateur d'états d'esprit et de logiques différentes : l'intendant agit en administrateur qui se veut efficace, la Chambre s'appuie sur un formalisme juridique qui s'embarrasse peu de considération sur le bon usage des revenus issus des octrois des villes. Finalement, l'intendant obtiendra gain de cause et ses ordonnancements de dépenses sur les octrois des villes seront acceptés par un arrêt du conseil enregistré à Nantes le 10 décembre $1711^{91}$. Quelques années plus tard, sous la Régence, lors des violents débats fiscaux autour des États de 1718, la Chambre semble au contraire mieux disposée envers le pouvoir de l'intendant et de la monarchie puisque Feydeau envisage même de l'utiliser contre ou à la place du Parlement ${ }^{92}$.

Entre ces deux moments de crise, 1711 et 1717/1718, se révèle peut-être l'intérêt de l'existence de la Chambre des comptes pour le pouvoir royal. La Chambre de par sa fonction de protection des finances royales se retrouve plus facilement aux côtés de la monarchie que les États ou le Parlement qui ont, dans une certaine mesure, un pouvoir d'ordonnancement et de répartition des dépenses publiques. De fait, si elle est parfois gênante pour l'intendant, elle peut aussi contrecarrer ou retarder les volontés des États ou du Parlement. De plus, les gens des comptes, de par leurs origines familiales, sont moins liés à la noblesse bretonne dominant les États que ne l'est le parlement. Ainsi il n'est probablement pas de bonne politique d'affaiblir trop la Chambre car on peut songer à l'utiliser pour contourner les blocages institutionnels générés par la mauvaise volonté des États, ou au moins menacer de le faire.

Cela n'empêche pas toutefois que les institutions provinciales - États, Parlement et Chambre des comptes -, puissent parfois se retrouver, dans

89. Cf. J. MeYER, J. BERENGER, La Bretagne en 1698 d'après le rapport de Béchameil de Nointel, Paris, Klincksieck, 1976, p. Iv-219.

90. Arch. dép. de Loire-Atlantique, C 186 à C 220, correspondance entre les intendants Ferrand puis Feydeau (1710-1721) et leur subdélégué à Nantes, Gérard Mellier, futur maire de la ville et général des finances (et à ce titre, membre de la Chambre).

91. Cf. H. FREVILLE, L'Intendance de Bretagne. 1689-1790 : essai sur l'histoire d'une intendance en pays d'États au XVIII siècle, s.l., Plihon, 1953, t. 1, p. 126-128.

92. Ibidem, t. 1, p. 190-193. 
leur opposition au bureau de finances par exemple. Mais, dans l'ensemble, l'existence de la Chambre permet aux agents de la monarchie de s'ingérer plus facilement dans les habitudes administratives bretonnes et de lézarder un front provincial ou un front de privilégiés.

Que conclure sur le tournant que représente le règne de Louis XIV? La Chambre est devenue une assemblée nombreuse, elle a fortement contribué aux finances royales par les créations d'offices, les prêts de toute sorte, les rachats d'offices ou les diverses taxes pour confirmation de noblesse qui touchent certains de ses membres. En son sein, les réelles fonctions de pouvoir, celles de premier président et de procureur général sont désormais passées aux mains des Becdelièvre et des La Tullaye qui les conserveront jusqu'à la Révolution, gage probable de sécurité et de stabilité pour l'institution, mais aussi indice possible d'un caractère routinier qui s'accroît. Mais l'évolution administrative de la monarchie a contribué à réduire ses attributions. Face aux exigences permanentes et au renforcement de l'absolutisme, la Chambre a payé son droit à survivre, à subsister. Mais, de toute évidence, ses fonctions sont de plus en plus formelles, voire superflues et on sait - quand on le veut - s'en passer.

\section{Le déclin du XVIII siècle}

Après ces changements nombreux étalés sur un demi-siècle, le XVIII siècle apparaît initialement comme très calme. En matière de personnel, la situation est même pratiquement figée : les seuls changements tiennent aux offices subalternes de greffier, payeur des gages ou substitut. Le règlement de 1681 ne connaîtra que des modifications ou des adaptations de détails sur le fonctionnement du parquet à cause de la création des offices de substituts, sur la limitation des délais de vérification des comptes ou sur le rôle de la chambre dans la vérification des comptes des nouveaux impôts du XVIII ${ }^{\mathrm{e}}$ siècle, comme le vingtième par exemple. Certes les conflits de compétence avec le Parlement, les États ou les municipalités urbaines existent toujours mais semblent moins aigus et plus facilement réglés. Bref, la Chambre des comptes semble paisible, coulant des jours tranquilles et routiniers sur les bords de l'Erdre!

L'observation des livres et minutes d'audience montre alors ce qui fait son quotidien : délivrance d'extraits anciens, enregistrement de lettres de noblesse ou de naturalité, vérification des comptes des villes, de la capitation ou des rentes sur les États de Bretagne ou préoccupation pour l'encombrement des armoires par les archives anciennes ou pour le délabrement de plus en plus marqué de son palais amenant à la reconstruction de la deuxième moitié du siècle.

Mais on y relève aussi, et de façon beaucoup plus sensible qu'auparavant, l'importance des querelles et conflits internes, l'extrême attention des officiers aux problèmes liés aux gages et aux épices et l'insistance de la Chambre à maintenir ses droits, ses prérogatives, sa position dans une hié- 
rarchie symbolique des honneurs et des fonctions où elle est de plus en plus menacée. Ce repli sur des querelles particulières et sur des préséances n'est sans doute pas de pur hasard. En fait, la Chambre apparaît de plus en plus dépouillée de fonctions réellement importantes et reléguée dans des activités formelles ou une fonction de conservation d'archives et de délivrance d'actes anciens ${ }^{93}$. L'installation permanente de l'intendant et le contrôle qu'il exerce sur les finances urbaines réduisent ses possibilités d'intervention dans ce domaine. La création régulière de nouveaux impôts, capitation, dixième, cinquantième, vingtième, limite l'importance proportionnelle des prélèvements anciens (fouages, impôts et billots...) sur lesquels elle avait un regard attentif, le déclin très marqué du Domaine royal parmi les ressources de l'État, de même que l'engagement d'une partie de ce domaine par le comte de Toulouse ou son aliénation au profit des États provinciaux, en multipliant les intermédiaires rendent son pouvoir de surveillance de plus en plus illusoire. Dès 1730, quand on se préoccupe à nouveau de réformer certains éléments du domaine royal, la tâche est confiée à un parlementaire, Dupont d'Oville, et non plus aux gens des comptes ${ }^{94}$. Le rôle croissant du parlement et des États après la création de la Commission intermédiaire en particulier, contribue aussi à marginaliser l'institution. Dès lors, l'esprit de corps et de défense se cristallise autour des manifestations les plus visibles d'un pouvoir de toute évidence déclinant. Réclamer la construction d'un nouveau palais, s'arc-bouter sur les privilèges de bancs dans la cathédrale de Nantes, sur la place de Nosseigneurs des Comptes dans les processions de la Fête Dieu, sur le calcul des épices lors des vérifications du compte des États, sur l'égalité des frais de voyages payés aux gens des comptes et à ceux du parlement lors de députation auprès de la Cour... sont autant d'éléments qui laissent l'impression aux gens des comptes (et l'espère-t-on, aux autres) qu'ils continuent à tenir leur place dans la hiérarchie sociale alors qu'en réalité ils ont laissé échapper (ou on leur a retiré de fait) une grande partie de leurs pouvoirs réels.

Ce déclin discret s'accompagne d'un changement dans les types de recrutement d'officiers et d'une modification de l'image de la Chambre dans la société. À Nantes, le décalage entre ces officiers, dont le rôle paraît de plus en plus secondaire, qui siègent jusqu'au milieu du siècle dans un palais en ruine, et les richesses accumulées par le commerce colonial est sans doute frappant. Un fils d'armateur ou de négociant peut-il être tenté par la Chambre des comptes alors que s'ouvrent à lui avec les fortunes coloniales des possibilités d'ascension sociale plus rapides et prestigieuses? Dans le reste de la province, les incessants conflits d'autorité avec le parlement et les États, les demandes pointilleuses de ses huissiers en tournées peu-

93. L'insistance mise sur l'intérêt pour le roi, pour la province, pour les municipalités et pour les particuliers de la conservation, de la protection et du classement des archives de la Chambre lors des opérations de déménagement et de reconstruction du palais est significative de cet état de fait

94. Le phénomène commence dès la fin du XVII ${ }^{\mathrm{e}}$ siècle. $C f$. Arch. dép. d'Ille-et-Vilaine, C 1903. 
vent-ils laisser de la Chambre une image attirante? On peut se le demander et les marques d'un certain irrespect pour l'institution paraissent plus fréquentes. C'est le procès intenté par le richissime négociant anobli Walsh contre un maître pour le titre de "messire ${ }^{95}$ ", c'est l'injure publique faite par un obscur bourgeois au procureur général ${ }^{96}$, ce sont les extrêmes difficultés des huissiers à se faire obéir et les quelques mentions négatives dans les cahiers de doléances.

Le rôle politique effacé de la Chambre dans la seconde moitié du XVIII ${ }^{\mathrm{e}}$ siècle ne contribue sans doute pas à augmenter son prestige. Parfois relativement proche du duc d'Aiguillon, en particulier quand celui-ci encourage les travaux pour la reconstruction de son palais, elle ne joue qu'un rôle très secondaire dans "l'affaire de Bretagne ", même si elle ne peut qu'embrasser la position des parlementaires bretons et des cours souveraines dans leur ensemble. Bien que certains de ses membres aient pu être tentés par le jansénisme, elle n'apparaît jamais au premier plan dans le combat contre les Jésuites. Son violent conflit avec la municipalité de Nantes autour de 1780 est très significatif des contradictions dans lesquelles elle est plongée. S'opposant sur des points de contrôle financier, mais aussi de marque de hiérarchie et de prestige social au corps de ville de la cité la plus riche et moderne de la province, elle ne peut guère qu'apparaître comme archaïque et rétrograde. Face aux réalités économiques, humaines et politiques, il est presque inconcevable que la monarchie prenne sa défense, quels que soient par ailleurs, les arguments juridiques qu'elle pourrait invoquer. Pour les mêmes raisons, tactiques, politiques et de réalisme administratif, les États de Bretagne, eux-mêmes parfois confrontés à la mauvaise volonté de la Chambre, ne peuvent non plus la suivre et seul le Parlement, au nom de la défense des cours souveraines lui apportera un soutien par ailleurs modéré. L'exil à Redon, même s'il ne se traduira pas dans les faits par un réel déménagement, achève sans doute de la déconsidérer aux yeux d'une partie de l'opinion. En fait, à l'inverse des combats du Parlement qui réussissent à regrouper autour de lui la majorité de l'opinion bretonne, cet affrontement l'isole encore plus. La Chambre apparaît plus comme le défenseur de ses propres privilèges, des prérogatives de quelques puissants que comme le bras actif d'une résistance face à un pouvoir extérieur envahissant. En s'en prenant au nom d'un légalisme ou d'un juridisme exacerbé à la municipalité nantaise, en exigeant un respect et une soumission surannée à une institution dont beaucoup ne comprennent plus les raisons d'être, la Chambre révèle en quelque sorte les contradictions internes du système politique de la monarchie finissante et son incapacité à choisir entre les formes traditionnelles du pouvoir et les choix rigoureux d'une administration au service de tous les sujets ${ }^{97}$.

95. Arch. dép. de Loire-Atlantique, B 152.

96. Idem, B 155, 156 et 170.

97. Sur toute cette affaire très peu évoquée par l'historiographie, voir les longs développements d'A. DuRAnd, La Chambre des comptes de Nantes, Nantes, Conseil général de Loire-Atlantique, 1976. 
L'épisode ne sera d'ailleurs pas sans conséquence. Les dernières avancées du pouvoir des États de Bretagne face à la monarchie dans les années 1780 se font en partie au détriment des prérogatives traditionnelles de la Chambre en matière de Domaine royal et de fiscalité. Si, en 1787 et 1788, elle s'associe vigoureusement aux protestations des cours souveraines contre les projets de réforme politiques et judiciaires, si elle envoie elle aussi des remontrances où perce de toute évidence l'esprit des Lumières, si elle est aussi victime de lits de justice pour l'enregistrement des édits, si nombre de ses membres figurent parmi les signataires des protestations nobiliaires de l'époque, si elle réclame comme d'autres la réunion d'États Généraux, elle n'en reste pas moins un acteur très secondaire des événements qui se jouent alors, image d'une réalité politique et institutionnelle d'un autre âge.

\section{RESUME}

Pendant les trois siècles de l'Ancien Régime, l'évolution de la Chambre des comptes reflète, au prix de quelques crises violentes et de mutations discrètes, les changements survenus dans l'histoire institutionnelle et politique de la province et du royaume. Alors que le nombre d'officiers s'accroît selon des rythmes et des modalités déjà connus ailleurs en France, les fonctions de la Chambre sont peu à peu délimitées et réduites. Les troubles de la seconde moitié du XVI ${ }^{\mathrm{e}}$ siècle puis le règne de Louis XIV marquent les temps forts de cette évolution qui conduit l'institution au rôle très secondaire qui sera le sien au $X V I I I^{\mathrm{e}}$ siècle.

\section{ABSTRACT}

During the three centuries of the Old Regime, the evolution of the Chambre des comptes reflects, at the price of some violent crises and discrete alterations, the changes which have occurred in the institutional and political history of the province and of the kingdom. Whereas the number of officers increases according to rates and processes which are already known, the functions of the Room are little by little delimited and reduced. The disorders of second half of the XVI ${ }^{\text {th }}$ century then the reign of Louis XIV represent the height of this evolution which leads the institution to a very secondary role during the XVIII th century. 
